\title{
UNIVERSAL VARIETIES OF DISTRIBUTIVE DOUBLE p-ALGEBRAS
}

\author{
by V. KOUBEK $\dagger$ and J. SICHLER $\dagger$
}

(Received 10 January, 1984)

1. Introduction. An algebra $\left(L ; \vee, \wedge,{ }^{*},{ }^{+}, 0,1\right)$ of type $(2,2,1,1,0,0)$ is a distributive double $p$-algebra provided $(L ; \vee, \wedge, 0,1)$ is a distributive $(0,1)$-lattice, and ${ }^{*},{ }^{+}$are unary operations of pseudocomplementation, or dual pseudocomplementation, respectively: the operation * satisfies $x \leqslant a^{*}$ if and only if $x \wedge a=0$, while $x \geqslant a^{+}$holds if and only if $x \vee a=1$.

A category $C$ is universal (or binding) if any full category of algebras is isomorphic to a full subcategory of $C$. In particular, see [10] or [18], for every monoid $M$ there is a proper class $K$ of pairwise non-isomorphic objects in $C$ such that $M$ is isomorphic to the endomorphism monoid $\operatorname{End}(X)$ for every $X \in K$; hence any binding class $C$ of algebras contains arbitrarily large members with endomorphism monoids isomorphic to $M$.

In [3] it was shown that the category $D P$ of all homomorphisms of distributive double p-algebras contains infinitely many non-isomorphic finite algebras $X_{i}$ with $\operatorname{End}\left(X_{i}\right) \cong M$ for any finite monoid $M$, and that there exist arbitrarily large double p-algebras with one-element endomorphism monoid. In the present paper we exhibit a finitely generated universal subvariety $V$ of $D P$; this strengthens the results of [3] and also answers a question, raised by Ervin Fried, of whether a finitely generated congruence distributive variety can be universal as a category. Since any nontrivial finitely generated variety of distributive double p-algebras contains a finite simple algebra [4], [6], it is natural to ask whether a variety generated by finitely many finite simple double p-algebras can be universal. We show that this is not the case; in fact, there are groups not representable as automorphism groups of algebras in such a variety.

A category $E$ is an iso-category if all morphisms of $E$ are invertible; thus the category Iso $(C)$ of all isomorphisms of any category $C$ is an iso-category. We say that a category $C$ is iso-universal if $\operatorname{Iso}(A)$ is isomorphic to a full subcategory of Iso $(C)$ for any full category $A$ of algebras. Consequently [18], if $C$ is an iso-universal category of algebras then, for any group $G$, there is a proper class $K$ of algebras in $C$ such that $\operatorname{Aut}(X) \cong G$ for every $X \in K$. In this terminology, we show that a finitely generated variety $V$ of double $\mathrm{p}$-algebras is iso-universal if and only if it contains a subdirectly irreducible algebra which is not simple, that is, if and only if $V$ is not a congruence permutable variety. On the other hand, the variety generated in DP by all simple algebras of range two is congruence permutable and iso-universal. We are thus led to the following modifications of Fried's original question.

$\dagger$ The support of NSERC is gratefully acknowledged.

Glasgow Math. J. 26 (1985) 121-131. 
Problem 1. Are there any iso-universal or universal finitely generated congruence permutable varieties of any type?

In connection with this question, it should be pointed out that congruence permutability and universality do not exclude each other in general: adding a new nullary operation to Heyting algebras constructed in [2] produces a universal congruence permutable variety.

Problem 2. Characterize finitely generated universal varieties of double p-algebras.

It is a pleasure to acknowledge stimulating and extensive correspondence with $\mathbf{R}$. Beazer on varieties of double $p$-algebras, as well as numerous fruitful discussions with $\mathbf{M}$. E. Adams concerning Priestley's duality.

2. Preliminaries. Priestley's duality [14], [15] for distributive lattices is used extensively throughout this paper. We recall briefly its basic properties; for details, the reader is referred to B. Davey [6], H. A. Priestley [14], [15], or to overview articles by B. Davey and D. Duffus [7], H. A. Priestley [17].

An ordered space $(X ; \tau, \leqslant)$ consists of a poset $(X ; \leqslant)$ and a topology $\tau$ on $X$. A subset $A$ of $X$ is decreasing if $x \leqslant a \in A$ implies $x \in A$ for every $x \in X$; increasing set is defined dually. An ordered space $(X ; \tau, \leqslant)$ is totally order disconnected if $x \neq y$ implies the existence of a clopen decreasing set $A \subseteq X$ with $x \in A$ and $y \notin A$ for any $x, y \in X$. Let $C T$ denote the category of all continuous order preserving maps between compact totally order disconnected spaces, and let $D$ be the category of all $(0,1)$-homomorphisms of distributive $(0,1)$-lattices.

Theorem $2.1(\mathrm{H}$. A. Priestley [14]). There is a contravariant isofunctor $\Delta: C T \rightarrow D$. The lattice $\Delta(X ; \tau, \leqslant)$ is the inclusion-ordered set of all clopen decreasing subsets of $(X ; \tau, \leqslant)$. For any $C T$-morphism $f: S \rightarrow S^{\prime}$ and every clopen decreasing subset $Y^{\prime}$ of $S^{\prime}$, the lattice homomorphism $\Delta(f)$ is given by $\Delta(f)\left(Y^{\prime}\right)=f^{-1}\left(Y^{\prime}\right)$.

Let $\operatorname{Min}(x)$ be the set of all minimal elements of $(X ; \leqslant)$ below $x \in X$ and, for any $A \subseteq X$, let $\operatorname{Min}(A)$ be the union $\cup(\operatorname{Min}(a): a \in A)$; define $\operatorname{Max}(x)$ and $\operatorname{Max}(A)$ dually. The sets $\operatorname{Min}(x)$ and $\operatorname{Max}(x)$ are nonvoid for any element $x$ of a compact totally order disconnected space $(X ; \tau, \leqslant)$, called also a Priestley space. For any $a \in X$, let $(a]=$ $\{x \in X: x \leqslant a\},[a)=\{x \in X: a \leqslant x\}$; given a subset $A$ of $X$, set $(A]=\bigcup((a]: a \in A)$ and $[A)=\bigcup([a): a \in A)$. Clearly $[A)=[\operatorname{Min}(A))$ for any decreasing subset $A$ of $X$, and, dually, $(A]=(\operatorname{Max}(A)]$ if $A$ is increasing. The following result describes the restriction of Priestley's duality to the category $D P$ of distributive double p-algebras [15]; see also [17] or [6].

Theorem $2.2(\mathrm{H}$. A. Priestley). If $S=(X ; \tau, \leqslant)$ is a Priestley space then $\Delta(S)$ lies in $D P$ if and only if $[A)$ is clopen for any clopen decreasing set $A$ of $S$, and $(B]$ is clopen for any clopen increasing set $B$ of $S$.

Let $f: S^{\prime} \rightarrow S$ be a CT-morphism with $\Delta(S), \Delta\left(S^{\prime}\right) \in D P$. Then $\Delta(f): \Delta(S) \rightarrow \Delta\left(S^{\prime}\right)$ is a $D P$-morphism if and only if $f(\operatorname{Min}(x))=\operatorname{Min}(f(x))$ and $f(\operatorname{Max}(x))=\operatorname{Max}(f(x))$ for every 
$x \in X$. Furthermore, $\Delta(f)$ is one-to-one (onto) if and only if $f$ is onto ( $f$ is a homeomorphism and order isomorphism of $S^{\prime}$ into $S$ ).

Next we recall a characterization of finite subdirectly irreducible distributive double p-algebras due to B. Davey [6].

TheORem 2.3 (B. Davey). Let $S=(X ; \tau, \leqslant)$ be a Priestley space. Then

(a) $\Delta(S)$ is a finite simple double $p$-algebra if and only if $(X ; \leqslant)$ is a finite connected poset such that $X=\operatorname{Min}(X) \cup \operatorname{Max}(X)$;

(b) $\Delta(S)$ is a finite subdirectly irreducible double $p$-algebra if and only if $(X ; \leqslant)$ is a finite connected poset and $X$ has at most one element outside $\operatorname{Min}(X) \cup \operatorname{Max}(X)$.

Given a finite simple distributive double p-algebra $F$, let $V(F)$ denote the subvariety of DP generated by all finite subdirectly irreducible algebras having $F$ as a homomorphic image. Theorems 2.2 and 2.3 combine to give Proposition 2.4 below.

Proposirion 2.4. The variety $V(F)$ is finitely generated.

The following sufficient condition for simplicity is easily obtained from Theorem 2.4 of B. Davey [6].

Propostrion 2.5. If $\Delta(X ; \tau, \leqslant) \in D P$ is such that $(X ; \leqslant)$ is a connected poset with $X=\operatorname{Min}(X) \cup \operatorname{Max}(X)$ then $\Delta(X ; \tau, \leqslant)$ is a simple distributive double $p$-algebra.

Let $L_{3}$ denote the category whose objects $(L ; a, b, c)$ consist of a distributive $(0,1)$-lattice and three constants $a, b, c \in L \backslash\{0,1\}$ satisfying $a \vee b=1>a \vee c, c<b$, and $a \wedge c=0<a \wedge b$; the morphisms of $L_{3}$ are all lattice homomorphisms preserving these three constants. It was shown in [1] that $L_{3}$ is a universal category. Since every lattice homomorphism preserving the three constants is a $(0,1)$-homomorphism, Priestley's duality can be used to reformulate Theorem 1.1 of [1] as follows.

Propostrion $2.6[1]$. The category $T^{3}$ of all Priestley spaces $X$ with three distinguished clopen decreasing sets $A, B, C$ satisfying $A \cap C=\varnothing, C \subseteq B, A \cup B=X$, and of all continuous order preserving maps $f:(X ; A, B, C) \rightarrow\left(X^{\prime} ; A^{\prime}, B^{\prime}, C^{\prime}\right)$ of $X$ into $X^{\prime}$ such that $f(A \backslash B) \subseteq A^{\prime} \backslash B^{\prime}, f(A \cap B) \subseteq A^{\prime} \cap B^{\prime}, f(B \backslash(A \cup C)) \subseteq B^{\prime} \backslash\left(A^{\prime} \cup C^{\prime}\right)$, and $f(C) \subseteq C^{\prime}$ is $d u-$ ally isomorphic to a universal category.

Any totally order disconnected space $(X ; \tau, \leqslant)$ whose topology $\tau$ has a subbase consisting of clopen decreasing sets and their complements (that is, clopen increasing sets) has an open base formed by sets of the form $C \cap D$ with $C$ clopen increasing and $D$ clopen decreasing; any such space will be called order regular. Clearly, any subspace of a Priestley space $S$ whose order is the restriction of that of $S$ is an order regular space; on the other hand, every compact order regular space is a Priestley space.

For an order regular space $(X ; \tau, \leqslant)$, let $K(X)$ be the Boolean algebra of all clopen sets of $(X ; \tau)$, and let $\beta X$ denote the set of all prime filters of $K(X)$. For every $W \in K(X)$ set $\operatorname{cl}(W)=\{a \in \beta X: W \in a\}$, and let $B=\{\operatorname{cl}(W): W \in K(X)\}$. It is easy to see that $B$ is closed under finite unions; there is a topology $\sigma$ of $\beta X$ having $B$ as its base for closed sets. 
Standard arguments show that $(\beta X ; \sigma)$ is a compact totally disconnected space containing $(X ; \tau)$ as a dense subspace, once every $x \in X$ is identified with the prime filter $p(x)=$ $\{W \in K(X): x \in W\}$ of $K(X)$. Every continuous map $f$ of $(X ; \tau)$ into a compact totally disconnected space $Y$ extends uniquely to a continuous map $g$ defined on $(\beta X ; \sigma)$. Define a relation $e \subseteq \beta X \times \beta X$ as the set of all pairs $(a, b)$ such that $b \in \operatorname{cl}(W)$ implies $a \in \operatorname{cl}(W)$ for every decreasing $W \in K(X)$. It is easily seen that $e$ is a preorder on $\beta X$. If $x, y \in X$ then $(x, y) \in e$ is equivalent to $x \leqslant y$ since $(X ; \tau, \leqslant)$ is totally order disconnected; furthermore, $(a, x),(x, a) \in e$ can hold only if $a=x$.

If $\varphi: \beta X \rightarrow \alpha X$ is an onto map such that $\varphi(a)=\varphi(b)$ if and only if $(a, b),(b, a) \in e$, then the $\varphi$-quotient $\leqslant$ of $e$ is a partial order and the quotient topology $\nu$ is compact and totally order disconnected with respect to $\leqslant$. Thus $(\alpha X ; \nu, \leqslant)$ is a Priestley space; its existence was first proved by L. Nachbin [13] (see also H. A. Priestley [14]). It is easy to show that the continuous extension $g$ of an order preserving continuous mapping $f$ of $(X ; \tau, \leqslant)$ into a Priestley space $Y$ has the form $g=h \circ \varphi$ for some continuous order preserving mapping $h$.

Proposition 2.7 (L. Nachbin, H. A. Priestley). For every order regular space $(X ; \tau, \leqslant)$ there exists a uniquely determined Priestley space $(\alpha X ; \nu, \leqslant)$ containing $(X ; \tau, \leqslant)$ as an ordered dense subspace such that every continuous order preserving mapping $f$ of $(X ; \tau, \leqslant)$ into a Priestley space $(Y ; \rho, \leqslant)$ extends to a continuous order preserving mapping $h:(\alpha X ; \nu, \leqslant) \rightarrow(Y ; \rho, \leqslant)$.

If $S_{i}=\left(X_{i} ; \tau_{i}, \leqslant_{i}\right)$ is a Priestley space for every $i \in I$, set $X$ to be the disjoint union of $\left(X_{i}: i \in I\right)$, and define $x \leqslant y$ iff $x \leqslant_{i} y$ for some $i \in I$. Equipped with the union topology $\tau$, the space $\sum\left(S_{i}: i \in I\right)=(X ; \tau, \leqslant)=S$ is order regular; the inclusion map $e_{i}$ of $X_{i}$ into $X$ is a homeomorphism of $S_{i}$ into $S$ and also an order embedding for every $i \in I$. Hence $e_{i}$ is also a continuous order embedding of $S_{i}$ into $\alpha S=(\alpha X ; \nu, \leqslant)$. If $Y$ is a Priestley space and $f_{i}: S_{i} \rightarrow Y$ is a continuous order preserving mapping for every $i \in I$, then the joint extension $f: S \rightarrow Y$ of all $f_{i}$ preserves order and is continuous. The continuous extension $h$ of $f$ from Proposition 2.7 preserves order and $h \circ e_{i}=f_{i}$ for every $i \in I$. Since $h$ is the only continuous map with the latter property, it follows from Theorem 2.1 that $\Delta(\alpha S)$ is isomorphic to the product $\Pi\left(\Delta\left(S_{i}\right): i \in I\right)$ of distributive lattices $\Delta\left(S_{i}\right)$. The product of double p-algebras is again a double p-algebra; this completes the proof of the claim below.

PropostTion 2.8. For any system $\left(\Delta\left(S_{i}\right): i \in I\right)$ of double p-algebras, the product $\Pi\left(\Delta\left(S_{i}\right): i \in I\right)$ is isomorphic to the dual of $\alpha \sum\left(S_{i}: i \in I\right)$.

3. A finitely generated universal variety. Theorem 4.6 of the next section shows that any universal variety of distributive double p-algebras must contain a subdirectly irreducible algebra which is not simple. The universal variety $V$ exhibited here will be generated by four finite subdirectly irreducible algebras whose quotients modulo their respective critical congruences are isomorphic to a simple algebra $F$.

To prove the universality of such a variety $V$, a full embedding $\Phi$ of the category $T^{3}$ 
described in Proposition 2.6 into the class of Priestley spaces dual to algebras in $V$ will be constructed as follows.

Let $T=(X ; A, B, C)$ be an object of $T^{3}$. Extending $X$ by the set $S=$ $\left\{a_{0}, c_{0}, p_{0}, q_{0}, a_{1}, b_{1}, p_{1}, q_{1}\right\}$ of eight isolated points preserves compactness. The order of the compact space $\Phi(T)=(X \cup S ; \tau, \leqslant)$ is defined by

(i) for any $x, y \in X, x \leqslant y$ in $\Phi(T)$ if and only if $x \leqslant y$ in $X$,

(ii) $\operatorname{Min}(\Phi(T))=\left\{a_{0}, c_{0}, p_{0}, q_{0}\right\}$ and $\operatorname{Max}(\Phi(T))=\left\{a_{1}, b_{1}, p_{1}, q_{1}\right\}$,

(iii) $\operatorname{Min}\left(a_{1}\right)=\left\{p_{0}, c_{0}\right\}, \operatorname{Max}\left(c_{0}\right)=\left\{a_{1}, q_{1}, b_{1}\right\}, \operatorname{Min}\left(b_{1}\right)=\left\{c_{0}, q_{0}, a_{0}\right\}, \operatorname{Max}\left(a_{0}\right)=\left\{b_{1}, p_{1}\right\}$, and $q_{0} \leqslant q_{1}$;

furthermore, for any $x \in X$,

(iv) $c_{0} \leqslant x$ (if and only if $x \in X \backslash C ; a_{0} \leqslant x$ if and only if $x \in X \backslash A$;

(v) $x \leqslant a_{1}$ if and only if $x \in A ; x \leqslant b_{1}$ if and only if $x \in B$.

It is easily verified that (i)-(v) indeed define a partial order on $X \cup S$; since the poset $X$ is convex in $\Phi(T)$, the ordered space $\Phi(T)$ is totally order disconnected. To see that $\Phi(T)$ is a space dual to a double p-algebra, in view of (ii) it suffices to observe that $\left[a_{0}\right) \cap X=X \backslash A,\left[c_{0}\right) \cap X=X \backslash C,\left[p_{0}\right) \cap X=\left[q_{0}\right) \cap X=\varnothing$ are clopen in $\Phi(T)$, and so are the sets $\left(a_{1}\right] \cap X=A,\left(b_{1}\right] \cap X=B$, and $\left(p_{1}\right] \cap X=\left(q_{1}\right] \cap X=\varnothing$.

The conditions (ii) and (iii) show that $(S ; \leqslant)$ is a connected poset with $S=$ $\operatorname{Min}(S) \cup \operatorname{Max}(S)$; by Theorem 2.3(a), $\Delta(S)$ is a simple double p-algebra. If $\Delta(\mathrm{g})$ is an endomorphism of $\Delta(S)$ then the finiteness of $S$ implies that $g$ is a bijection of $S$ onto itself. Since $p_{0} \in S$ is the only minimal element of $S$ with exactly one maximal element above it, $g\left(p_{0}\right)=p_{0}$ follows by Theorem 2.2. Furthermore, $g\left(c_{0}\right)=c_{0}$ because $|\operatorname{Max}(s)|=3$ if and only if $s=c_{0}$. Arguments dual to these show that $g\left(p_{1}\right)=p_{1}$ and $g\left(b_{1}\right)=b_{1}$. From $\operatorname{Min}\left(p_{1}\right)=\left\{a_{0}\right\}, \operatorname{Max}\left(p_{0}\right)=\left\{a_{1}\right\}$ it follows that $g\left(a_{0}\right)=a_{0}, g\left(a_{1}\right)=a_{1}$, respectively. Now $g\left(q_{i}\right)=q_{i}$ for $i=0,1$ since $g$ permutes $\operatorname{Max}(S), \operatorname{Min}(S)$. Thus the algebra $\Delta(S)$ is rigid, that is, its endomorphism monoid is trivial.

Let $f:(X ; A, B, C) \rightarrow\left(X^{\prime} ; A^{\prime}, B^{\prime}, C^{\prime}\right)=T^{\prime}$ be a morphism of $T^{3}$, see Proposition 2.6. Using Theorem 2.2 it is routine to verify that the extension $\Phi(f): \Phi(T) \rightarrow \Phi\left(T^{\prime}\right)$ of $f$ by the identity of $S$ is a dual of a double p-algebra homomorphism. This also shows that $\Phi$ is a faithful functor.

To show that the image of $\Phi$ is a full subcategory in the category of all Priestley spaces dual to double p-algebras, let $g: \Phi(T) \rightarrow \Phi\left(T^{\prime}\right)$ be such that $\Delta(g) \in D P$. Since $\operatorname{Min}(\Phi(T)) \cup \operatorname{Max}(\Phi(T))=S=\operatorname{Min}\left(\Phi\left(T^{\prime}\right)\right) \cup \operatorname{Max}\left(\Phi\left(T^{\prime}\right)\right)$, the restriction of $\mathrm{g}$ to $S$ is an endomorphism of $S$; since $S$ is rigid, g preserves $S$ pointwise. From (iii), (iv), and (v) it follows that $\operatorname{Min}(z)=\left\{c_{0}\right\}$ and $\operatorname{Max}(z)=\left\{a_{1}\right\}$ hold simultaneously for any $z \in \Phi(T)$ if and only if $z \in X \backslash B=A \backslash B$. Thus $g(A \backslash B) \subseteq A^{\prime} \backslash B^{\prime}$ by Theorem 2.2. An element $z$ of $A \cap B$ is characterized by $\operatorname{Min}(z)=\left\{c_{0}\right\}$ and $\operatorname{Max}(z)=\left\{a_{1}, b_{1}\right\}$, so that $g(A \cap B) \subseteq A^{\prime} \cap B^{\prime}$. Elements of $X \backslash(A \cup C)=B \backslash(A \cup C)$ are singled out as those with $\operatorname{Min}(z)=\left\{a_{0}, c_{0}\right\}$, $\operatorname{Max}(z)=\left\{b_{1}\right\}$, while $C$ is the set of all $z$ satisfying $\operatorname{Min}(z)=\left\{c_{0}\right\}$ and $\operatorname{Max}(z)=\left\{b_{1}\right\}$; the inclusions $g(B \backslash(A \cup C)) \subseteq B^{\prime} \backslash\left(A^{\prime} \cup C^{\prime}\right)$ and $g(C) \subseteq C^{\prime}$ thus follow analogously to the previous two cases. Proposition 2.6 concludes the proof of the claim below.

LEMMA 3.1. The full subcategory of DP determined by double p-algebras dual to spaces $\Phi(T)$ with $T \in T^{3}$ is universal. 
For any $T \in T^{3}$, the subspace $\operatorname{Min}(\Phi(T)) \cup \operatorname{Max}(\Phi(T))=S$ of $\Phi(T)$ represents a finite simple double p-algebra $F$; in fact, $F$ is the quotient of $\Delta(\Phi(T))$ modulo the determination congruence $\delta$, defined by $x \delta y$ if and only if $x^{*}=y^{*}$ and $x^{+}=y^{+}$, see R. Beazer [4]. Let $Q(F)$ denote the class of all algebras $A$ whose quotient by the determination congruence is isomorphic to $F$, or, equivalently, such that the dual $X$ of $A$ satisfies $\operatorname{Min}(X) \cup$ $\operatorname{Max}(X)=S$. To show that the class $\Phi\left(T^{3}\right)$ is contained in a finitely generated variety, we need the following claim.

LEMMA 3.2. If $F$ is a finite simple double $p$-algebra and $B \in Q(F)$ then $B$ is a subdirect product of subdirectly irreducible members of $Q(F)$.

Proof. Let $Y, S$ be the dual spaces of $B, F$, respectively. For any $y \in$ $Y \backslash(\operatorname{Min}(Y) \cap \operatorname{Max}(Y))$, the subspace $S(y)=S \cup\{y\}$ represents a finite subdirectly irreducible double p-algebra $A(y)$, see Theorem 2.3. From Theorem 2.2, it follows that $A(y) \in$ $Q(\Delta(S))$. The dual $\Delta\left(\xi_{y}\right)$ of the embedding $\xi_{y}: S(y) \rightarrow Y$ is a double p-algebra homomorphism for every $y \in Y \backslash S$. Since the embeddings $\xi_{y}$ are collectively onto, the algebra $\Delta(Y)$ is embedded into the direct product of algebras $\Delta(S(y))=A(y) \in Q(F)$.

Lemmata 3.1 and 3.2 combine as follows.

THEOREM 3.3. There exists a finitely generated universal variety of distributive double p-algebras, namely the variety $V(F)$ generated by all subdirectly irreducible algebras whose quotient modulo the critical congruence is isomorphic to the finite simple distributive double p-algebra $F$ represented by the poset $S$ defined by (i)-(iii) above.

It is easy to see that the four subdirectly irreducible algebras represented by finite posets containing $S \subseteq \Phi(T)$ whose respective nonextremal elements represent the relative order of $A \backslash B, A \cup B, B \backslash(A \cup C)$, and of $C$ to elements of $S$ already generate a variety containing the class $\Phi\left(T^{3}\right)$. Furthermore, every $\Phi(T)$ is dual to an algebra of range three, that is, $V(F)$ satisfies the identity $x^{4\left(+^{*}\right)}=x^{3\left(+^{*}\right)}$.

4. Iso-universal varieties. To investigate iso-universality of varieties generated by simple algebras we consider compactifications of split spaces, defined as order regular spaces $(X ; \tau, \leqslant)$ such that $X=\operatorname{Min}(X) \cup \operatorname{Max}(X)$ and both $m=\operatorname{Min}(X) \backslash \operatorname{Max}(X)$ and $M=\operatorname{Max}(X) \backslash \operatorname{Min}(X)$ are $\tau$-clopen.

LeMmA 4.1. If $S=(X ; \tau, \leqslant)$ is a split space then $\alpha S=\beta S$ is a compact split space with $\mathrm{cl}(\operatorname{Max}(X) \backslash \operatorname{Min}(X))=\operatorname{Max}(\beta S) \backslash \operatorname{Min}(\beta S)$ and $\operatorname{cl}(\operatorname{Min}(X) \backslash \operatorname{Max}(X))=\operatorname{Min}(\beta S) \backslash \operatorname{Max}(\beta S)$.

Proof. The space $S$ is a disjoint union of clopen sets $M, m$, and $I=\operatorname{Max}(X) \cap \operatorname{Min}(X)$. Suppose that $a \neq b$ and $(a, b) \in e$. If $m \in b$ then, for any $Z \in b$, the set $Z \cap m \in b$ is decreasing, so that $(a, b) \in e$ implies $Z \cap m \in a$. Thus $b \subseteq a$; since $a, b$ are prime filters, a contradictory $a=b$ is obtained. Therefore $m \notin b$, and, similarly, $I \notin b$; it follows that $M \in b$. Since $(a, b) \in e$ if and only if $W \in b$ for any clopen increasing $W \in a$, an argument dual to the previous one shows that $m \in a$. Thus $e$ is a partial order containing no three-element chain and, consequently, $\alpha S=\beta S$ is a compact split space such that $\operatorname{Max}(\beta S) \backslash \operatorname{Min}(\beta S) \subseteq$ 
$\operatorname{cl}(M)$ and $\operatorname{Min}(\beta S) \backslash \operatorname{Max}(\beta S) \subseteq \operatorname{cl}(m)$. Since $\operatorname{cl}(\operatorname{Min}(X))=\operatorname{Min}(\beta S)$ and $\operatorname{cl}(\operatorname{Max})(X))=$ $\operatorname{Max}(\beta S)$, from the definition of $M$ and $m$ it follows that $\operatorname{cl}(M) \subseteq \operatorname{Max}(\beta S) \backslash \operatorname{Min}(\beta S)$ and $\operatorname{cl}(m) \subseteq \operatorname{Min}(\beta S) \backslash \operatorname{Max}(\beta S)$.

The following claim describes the order of $\beta S$.

LEMMA 4.2. If $S=(X ; \tau, \leqslant)$ is a discrete split space then, for any $x \in X$ and $a \in \beta X \backslash X$, $x \leqslant a$ if and only if $x \in \operatorname{Min}(X)$ and $\operatorname{Max}_{S}(x) \in a$. In particular, if $\operatorname{Max}_{S}(x)$ is finite then $\operatorname{Max}_{\beta X}(x) \subseteq X$ for every $x \in X$.

Proof. If $Z \in a$ is decreasing and $\operatorname{Max}_{S}(x) \in a$, then $Z \cap \operatorname{Max}_{S}(x) \in a$ is nonvoid and hence $x \in Z$; thus $x \leqslant a$. Conversely, $[x) \in a$ follows from $x \leqslant a$. Since $x, a$ are distinct, $\operatorname{Max}_{S}(X) \in a$ and $x \in \operatorname{Min}_{S}(X)$ by Lemma 4.1, so that $\operatorname{Max}_{S}(x)=[x) \cap \operatorname{Max}(X) \in a$.

Let $(V, E)$ be an undirected graph, that is, let the set $E$ of edges consist of two-element subsets of the vertex set $V$. The graph $(V, E)$ will be connected: for every pair $v, w \in V$ there exist edges $e_{0}, \ldots, e_{n}$ such that $v \in e_{o}, w \in e_{n}$, and $e_{i} \cap e_{i+1} \neq \varnothing$ for $i=0, \ldots, n-1$.

For a connected graph $(V, E)$ set $X=V \cup E \cup\{p, q\}$, where $p, q$ are distinct elements not contained in $V \cup E$. Define a partial order on $X$ by requiring $\operatorname{Max}(X)=E \cup\{q\}$, $\operatorname{Min}(X)=V \cup\{p\}, v \leqslant e$ if and only if $v \in e, v \leqslant q$ for every $v \in V$, and $p \leqslant e$ for every $e \in E$. If $\delta$ is the discrete topology then $S=(X ; \delta, \leqslant)$ is a split discrete space whose order is connected. Set $\Phi(V, E)=\alpha S$. Then $\Phi(V, E)$ is a Priestley space, see Proposition 2.7; by Lemma 4.1, $\Phi(V, E)$ is defined on the set $\beta X$ of all ultrafilters on $X$. If $\operatorname{Max}(X) \in a$ and $\{q\} \in a$ then $a \in X$; otherwise $E \in a$, and $p \leqslant a$ follows from $E=\operatorname{Max}(p)$ by Lemma 4.2. In particular, $a \in \operatorname{Max}(\alpha S) \backslash \operatorname{Min}(\alpha S)$. If, on the other hand, $\operatorname{Max}(X)$ does not lie in $a$, then $\operatorname{Min}(X)=X \backslash \operatorname{Max}(X) \in a$. If $a \in \beta X \backslash X$ then $V=\operatorname{Min}(X) \backslash\{p\} \in a$, and $a \leqslant q$ is obtained from a statement dual to that of Lemma 4.2. Thus $a \in \operatorname{Min}(\alpha S) \backslash \operatorname{Max}(\alpha S)$ in this case. We see that $\alpha X$ is a compact split space with connected order.

To show that $\Phi(V, E)$ is a space dual to a simple double p-algebra, the claim below is needed, see Theorem 2.2 .

LEMMA 4.3. If the compactification $(\beta X ; \sigma, \leqslant)$ of $S=(X ; \tau, \leqslant)$ is partially ordered, and if the smallest increasing subset $\uparrow Y$ of $X$ containing $Y$ is $\tau$-clopen for any clopen decreasing $Y \subseteq X$, then $[C$ ) is $\sigma$-clopen for every $\sigma$-clopen decreasing $C \subseteq \beta X$.

Proof. Clearly $C \cap X \subseteq \uparrow(C \cap X) \subseteq[C)$, where $\uparrow(C \cap X)$ is $\tau$-clopen by hypothesis; since $[C)$ is closed in any Priestley space, $\operatorname{cl}(C \cap X) \subseteq \operatorname{cl}(\uparrow(C \cap X)) \subseteq[C)$. From the definition of $\leqslant$ on $\beta X$ it is clear that $\mathrm{cl}(\uparrow(C \cap X))$ is an increasing set; since $C=\operatorname{cl}(C \cap X)$, the claim follows because $\{C$ ) is the smallest increasing set containing $C$ and $\operatorname{cl}(Z)$ is $\sigma$-clopen for any $\tau$-clopen $Z \subseteq X$.

Lemma 4.3 and its dual show that $\Phi(V, E)$ represents a double p-algebra. Since $\Phi(V, E)$ is an order connected split space, the double p-algebra represented by $\Phi(V, E)$ is simple for any connected undirected graph $(V, E)$ by Proposition 2.5 .

Let $f:(V, E) \rightarrow\left(V^{\prime}, E^{\prime}\right)$ be a graph isomorphism, that is, a bijection of $V$ onto $V^{\prime}$ such that $\{v, w\} \in E$ if and only if $\{f(v), f(w)\} \in E^{\prime}$. The extension $f^{\prime}$ of $f$ to $V \cup E \cup\{p, q\}$ 
by $f^{\prime}(p)=p, f^{\prime}(q)=q$, and $f^{\prime}\{v, w\}=\{f(v), f(w)\}$ for all $\{v, w\} \in E$ is an order preserving isomorphism of $S=(X ; \delta, \leqslant)$ onto $S^{\prime}$. By Proposition 2.7 , there is a unique order preserving homeomorphism $\Phi(f)=\beta\left(f^{\prime}\right)$ of $\Phi(V, E)$ onto $\Phi\left(V^{\prime}, E^{\prime}\right)$. It is easily seen that $\Phi(f)(\operatorname{Max}(a))=\operatorname{Max}(\Phi(f)(a))$ and $\Phi(f)(\operatorname{Min}(a))=\operatorname{Min}(\Phi(f)(a))$ for every $a \in \beta X$. Clearly, $\Phi$ is a one-to-one functor of the iso-universal category Iso $(G)$ of all isomorphisms of connected graphs with more than two vertices $[8]$ into the category of Priestley spaces dual to the variety $Q$ generated by all simple double p-algebras $\Phi(V, E)$. Since the dual of an iso-universal category is iso-universal itself, the iso-universality of $Q$ will be demonstrated once it is shown that every order preserving homeomorphism $g$ of $\Phi(V, E)$ onto $\Phi\left(V^{\prime}, E^{\prime}\right)$ has the form $g=\Phi(f)$ for some invertible $f:(V, E) \rightarrow\left(V^{\prime}, E^{\prime}\right)$.

Let $g: \Phi(V, E) \rightarrow \Phi\left(V^{\prime}, E^{\prime}\right)$ be an order preserving continuous invertible mapping. A singleton set $\{a\} \in \beta X$ is $\sigma$-clopen if and only if $a \in X=V \cup E \cup\{p, q\}$; the mapping $g$ also preserves maximality and minimality. Hence $g(E \cup\{q\})=E^{\prime} \cup\{q\}$ and $g(V \cup\{p\})=$ $V^{\prime} \cup\{p\}$. The minimal elements of $\beta X$ below any $e=\{v, w\} \in E$ are just $v$ and $w$, by the dual of Lemma 4.2; if $g(e)=q$ then $\operatorname{Min}(q) \supseteq V^{\prime}$ can have only two elements. For $|V|$, $\left|V^{\prime}\right|>2$. we thus conclude $g(E)=E^{\prime}$ and $g(q)=q$. Since $p$ is the only minimal element not below $q$ in either space, $g(p)=p$ and $g(V)=V^{\prime}$ follow. Let $f$ denote the restriction of $g$ to the set $V$. If $\{v, w\} \in E$ then $g\{v, w\} \in E^{\prime}$ is an upper bound of $\{f(v), f(w)\} \subseteq V^{\prime}$, and $g\{v, w\}=\{f(v), f(w)\}$ follows by the definition of the order. Therefore the restriction of $g$ to $(X ; \delta, \leqslant)$ coincides with $f^{\prime}$ for some isomorphism $f$ of $(V, E)$ onto $\left(V^{\prime}, E^{\prime}\right)$; hence $g=\beta\left(f^{\prime}\right)=\Phi(f)$ as was to be shown.

Let us consider the variety $Q$ generated by all algebras $\Phi(V, E)$ in more detail. Recall that, for $c \in \Delta(T) \in D P$ represented by the clopen decreasing set $C$, the pseudocomplement $c^{*}$ of $c$ is represented by $C^{*}=T \backslash[C)$ and the dual pseudocomplement $c^{+}$of $c$ corresponds to $C^{+}=(T \backslash C]$. If $C$ is a proper clopen decreasing subset of $T=\Phi(V, E)=\beta X$, then the clopen set $(T \backslash C) \cap \operatorname{Max}(T)$ is nonvoid and hence it contains some $x \in \operatorname{Max}(X)=$ $E \cup\{q\}$. Consequently there is $v \in V$ such that $v \in C^{+}=(T \backslash C]$. Since $(V, E)$ is a connected graph, there exists some $e \in E$ in $\left[C^{+}\right)$; note that also $q \in\left[C^{+}\right)$. The set $C^{+*}=T \backslash\left\{C^{+}\right)$thus contains neither $e$ nor $q$, so that $\{e, q\} \subseteq\left(T \backslash C^{+*}\right]=C^{+*+}$. Every minimal element of $T$ is below $e$ or below $q$; thus $\operatorname{Min}(T) \subseteq C^{+*+}$, and $C^{+*+*}=\varnothing$ follows. Hence, in every generating algebra $\Phi(V, E)$ of $Q$, the polynomial $p(x)=x^{+*+*}=x^{2(+*)}$ satisfies $p(1)=1$ and $p(x)=0$ for all $x<1$. Consequently, $p(x)^{+*}=p(x)$, that is, every algebra of $Q$ is of range two.

Lemma 4.4 (R. Beazer [5]). Any variety $V$ of distributive double p-algebras generated by a class of simple algebras is congruence permutable.

Proof. The determination congruence on any simple algebra must be trivial, that is to say, every simple distributive double p-algebra is regular. Regular algebras form a subvariety $R$ of $D P$, see J. Varlet [19]. Since $R$ is congruence permutable [4], so is its subvariety $V$.

Lemma 4.4 and the arguments presented earlier combine as follows.

THEOREM 4.5. The class of all simple distributive double p-algebras of range two, and 
hence also the congruence permutable variety $S(2)$ generated by simple algebras of range two are iso-universal.

Let $V$ be a finitely generated subvariety of $D P$. If $V$ has only simple algebras in its generating set then $V$ is congruence permutable by Lemma 4.4. Therefore any finitely generated variety $V$ which is not congruence permutable must contain a finite subdirectly irreducible algebra $T$ which is not simple. The dual of $T$ is a finite connected poset $D$ which is the disjoint union of $\operatorname{Max}(D), \operatorname{Min}(D)$, and a singleton $\{d\}$, see Proposition 2.3. Clearly, the three-element chain $C_{3}=\{0, c, 1\}$ is a quotient of $D$ via an onto map whose dual is a one-to-one homomorphism of $\Delta\left(C_{3}\right)$ into $\Delta(T)$. Hence the four-element chain $\Delta\left(C_{3}\right)$ is a subdirectly irreducible algebra in $V$; as a result, $V$ contains the variety $S^{2}$ of distributive double Stone algebras, see T. Katriňák [12]. The variety $S^{2}$ contains the five-element chain which, as a double p-algebra, is not congruence permutable. This establishes the equivalence of (i), (ii), and (iii) in Theorem 4.6 below.

THEOREM 4.6. The following conditions are equivalent for any finitely generated variety $V$ of distributive double p-algebras:

(i) $V$ is not congruence permutable,

(ii) $V$ contains a subdirectly irreducible algebra which is not simple,

(iii) the variety $S^{2}$ of distributive double Stone algebras is contained in $V$,

(iv) $V$ is iso-universal.

To show that (iii) implies (iv), define $\Psi(V, E)$ as the disjoint union $\Phi(V, E) \cup\{0,1\}$, where 0,1 are isolated points such that $0 \leqslant z \leqslant 1$ for all $z$ in $\Psi(V, E)$. It is clear that $\Psi(V, E)$ is a dual of a double p-algebra for any graph $(V, E)$, and that, for every graph isomorphism $f$, the mapping $\Psi(f)$ defined as the extension of $\Phi(f)$ by the identity mapping of $\{0,1\}$ is order preserving, continuous, and invertible. Conversely, any invertible order preserving homeomorphism $g: \Psi(V, E) \rightarrow \Psi\left(V^{\prime}, E^{\prime}\right)$ satisfies $g(\Phi(V, E))=\Phi\left(V^{\prime}, E^{\prime}\right)$ and preserves 0 and 1 ; thus $g \uparrow \Phi(V, E)=\Phi(f)$ for some graph isomorphism as in Theorem 4.5 and, as a result, $g=\Psi(f)$. From Lemma 3.2, it follows that $\Delta \circ \Psi$ maps the category Iso( $G)$ into the variety $S^{2}$ generated by the non-simple subdirectly irreducible fourelement chain $\Delta\left(C_{3}\right)$. Thus (iii) implies (iv).

To prove the converse implication, let $S$ be a finite set of finite simple double p-algebras generating a variety $V$; without a loss of generality we may assume that $S$ is hereditary. Translated by the duality, $S$ is represented by a set $F=\left\{Y_{1}, \ldots, Y_{n}\right\}$ of finite connected posets with discrete topology such that $Y=\operatorname{Min}(Y) \cup \operatorname{Max}(Y)$ for every $Y \in F$. Since $S$ is hereditary, $F$ is closed under quotients; apart from the one-element poset, any $Y \in F$ is a disjoint union of $\operatorname{Max}(Y)$ and $\operatorname{Min}(Y)$. Any algebra from $V$ is then dual to a quotient $T$ of some Priestley space $\alpha \sum\left(X_{i}: i \in I\right)$, where every $X_{i}$ is a finite poset isomorphic to a member of $F$, see Propositions 2.2 and 2.8.

To be more definite, let $k: I \rightarrow\{1, \ldots, n\}$ be an arbitrary mapping, and let $S$ be the union order on the set $X=\bigcup\left(\{i\} \times Y_{k(i)}: i \in I\right)$. Then $S$ is a split space; by Lemma 4.1 , the dual $\alpha S$ of the product is the split space $\beta S$ of all ultrafilters on $X$; order components of $\beta S$ are completely contained either in $S$ or in $\beta S \backslash S$ by Lemma 4.2. For every proper 
ultrafilter $a \in \beta X$ there is exactly one $j \in\{1, \ldots, n\}$ such that $k^{-1}\{j\} \in a$. The set $k^{-1}\{j\}$ is a disjoint union of finitely many sets of the form $k^{-1}\{j\} \times\{y\}$ with $y \in Y_{j}$. Thus every $a \in \beta X$ uniquely determines $j \in\{1, \ldots, n\}$ and $y \in Y_{j}$ such that $a \in \operatorname{cl}\left(k^{-1}\{j\} \times\{y\}\right)$. Since $\operatorname{cl}\left(X^{\prime}\right)$ properly contains $X^{\prime} \subseteq X$ whenever $X^{\prime}$ is infinite, it is easily seen that any order component of $\beta S \backslash S$ containing some $a \in \operatorname{cl}\left(k^{-1}\{j\}\right)$ is isomorphic to $Y_{j}$. If $T$ is a Priestley space representing a subalgebra of $\Pi\left(\Delta\left(Y_{k(i)}\right): i \in I\right)$ then every order component of $T$ is a quotient of an order component in $\beta S$; as a result, the cardinalities of order components of $T$ are finite and bounded. Let $\theta$ be an equivalence on $T$ consisting of all pairs $(t, u)$ such that $t, u \in Q$ for some order component $Q$ of $T$, and let $B$ be the quotient of $T$ modulo $\theta$. The space $B$ is clearly Boolean; let $h: T \rightarrow B$ denote the onto map with $\operatorname{Ker}(h)=\boldsymbol{\theta}$.

If $\operatorname{Aut}(T)$ is the group of all order preserving continuous invertible maps of $T$ into itself, then $g(Q)$ is an order component of $T$ for any order component $Q$ and any $g \in \operatorname{Aut}(T)$. Assume that $\operatorname{Aut}(T)$ contains an element $g$ of a prime order $p>|Y|$ for every $Y \in F$. For some $t \in T$ the elements $t_{r}=g^{r}(t)$ with $r \in p=\{0,1, \ldots, p-1\}$ are pairwise distinct, so that there are disjoint order components $Q_{0}, \ldots, Q_{p-1}$ of $T$ such that $g\left(Q_{r}\right)=Q_{r+1}$ (where the addition is performed modulo $p$ ) for all $r \in p$. Since the image $g(Q)$ of any order component $Q$ is order connected, there is an automorphism $g^{\prime}$ of $B$ satisfying $g^{\prime} \circ h=h \circ g$; if $\left\{b_{r}\right\}=h\left(Q_{r}\right)$, then $g^{\prime}\left(b_{r}\right)=b_{r+1}$ and the elements $b_{0}, \ldots, b_{\mathrm{p}-1}$ of $B$ are distinct. Since $B$ is totally disconnected there exists a clopen set $A_{0}$ such that $\left\{b_{r}: r \in p\right\} \cap A_{0}=\left\{b_{0}\right\}$; clearly, $b_{s} \in A_{r}=\left(g^{\prime}\right)^{r}\left(A_{0}\right)$ if and only if $s=r$. The sets $B_{r}=$ $A_{r} \backslash \cup\left(A_{s}: s \neq r\right)$ are pairwise disjoint, clopen, and such that $g^{\prime}\left(B_{r}\right)=B_{r+1}$ and $b_{r} \in B_{r}$ for every $r \in p$. Set $T_{r}=h^{-1}\left(B_{r}\right)$; the clopen sets $T_{r}$ are pairwise disjoint and $g\left(T_{r}\right)=T_{r+1}$ for all $r \in p$. For any permutation $\pi$ of $\{0, \ldots, p-1\}$ set $g_{\pi}(t)=g^{\pi(r)-r}(t)$ whenever $t \in T_{r}$, $g_{\pi}(t)=t$ for all $t \in T$ outside $T_{0} \cap \ldots \cap T_{\mathrm{p}-1}$. It is routine to verify that $g_{\pi} \in \operatorname{Aut}(T)$; thus $\operatorname{Aut}(T)$ contains the symmetric group $\operatorname{Sym}(p)$ for any element $g \in \operatorname{Aut}(T)$ of sufficiently large prime order $p$. Therefore the variety $V$ is not iso-universal. This completes the proof of Theorem 4.6.

\section{REFERENCES}

1. M. E. Adams, V. Koubek and J. Sichler, Homomorphisms and endomorphisms of distributive lattices, to appear in Houston J. Math.

2. M. E. Adams, V. Koubek and J. Sichler, Endomorphisms and homomorphisms of Heyting algebras, to appear in Algebra Universalis.

3. M. E. Adams and J. Sichler, Endomorphism monoids of distributive double p-algebras, Glasgow Math. J. 20 (1979), 81-86.

4. R. Beazer, The determination congruence on double p-algebras, Algebra Universalis 6 (1976), 121-129.

5. R. Beazer, Personal communication (1983).

6. B. Davey, Subdirectly irreducible distributive double p-algebras, Algebra Universalis 8 (1978), 73-88.

7. B. A. Davey and D. Duffus, Exponentiation and duality, Ordered Sets, NATO Advanced Study Institutes Series 83 (D. Reidel, 1982), 43-95.

8. Z. Hedrlín and A. Pultr, On rigid undirected graphs, Canad. J. Math. 18 (1966), 1237-1242. 
9. Z. Hedrlin and A. Pultr, On full embeddings of categories of algebras, Ilinois J. Math. 10 (1966), 392-406.

10. Z. Hedrlín and J. Sichler, Any boundable binding category contains a proper class of mutually disjoint copies of itself, Algebra Universalis 1 (1971), 97-103.

11. B. Jónsson, Algebras whose congruence lattices are distributive, Math. Scand. 21 (1967), $110-121$

12. T. Katriňák, The injective double Stone algebras, Algebra Universalis 4 (1974), 259-267.

13. L. Nachbin, Topology and Order (Van Nostrand, 1965).

14. H. A. Priestley, Representation of distributive lattices by means of ordered Stone spaces, Bull. London Math. Soc. 2 (1970), 186-190.

15. H. A. Priestley, Ordered topological spaces and the representation of distributive lattices, Proc. London Math. Soc. (3) 24 (1972), 507-530.

16. H. A. Priestley, The construction of spaces dual to pseudocomplemented distributive lattices, Quart. J. Math. Oxford Ser. (2) 26 (1975), 215-228.

17. H. A. Priestley, Ordered sets and duality for distributive lattices, Proc. Conf. on Ordered Sets and their Applications, Lyon (1982), to appear.

18. A. Pultr and V. Trnková, Combinatorial, Algebraic and Topological Representations of Groups, Semigroups and Categories (North-Holland, 1980).

19. J. Varlet, A regular variety of type (2, 2, 1, 1, 0, 0), Algebra Universalis 2 (1972), 218-223.

\section{MFF KU}

Malostranské nÁm. 25

Praha 1

Czechoslovakia

Department of Mathematics

UNIVERSITY OF MANITOBA

WINNIPEG, MANITOBA

CANADA 